\title{
Low-Frequency Stability Analysis of Periodic Traveling-Wave Solutions of Viscous Conservation Laws in Several Dimensions
}

\author{
Myunghyun Oh and Kevin Zumbrun
}

\begin{abstract}
We generalize the work of Oh \& Zumbrun and Serre on spectral stability of spatially periodic traveling waves of systems of viscous conservation laws from the one-dimensional to the multi-dimensional setting. Specifically, we extend to multi-dimensions the connection observed by Serre between the linearized dispersion relation near zero frequency of the linearized equations about the wave and the homogenized system obtained by slow modulation (WKB) approximation. This may be regarded as partial justification of the WKB expansion; an immediate consequence is that hyperbolicity of the multi-dimensional homogenized system is a necessary condition for stability of the waves. As pointed out by Oh \& Zumbrun in one dimension, the description of the low-frequency dispersion relation is also a first step in the determination of time-asymptotic behavior.
\end{abstract}

Keywords. Stability, WKB, periodic traveling-waves

Mathematics Subject Classification (2000). Primary 35L65, secondary 34L05

\section{Introduction}

Nonclassical viscous conservation laws arising in multiphase fluid and solid mechanics exhibit a rich variety of traveling wave phenomena, including homoclinic (pulse-type) and periodic solutions along with the standard heteroclinic (shock, or front-type) solutions. Here, we investigate stability of periodic traveling waves: specifically, the spectrum of the linearized operator about the wave. Our main result generalizes the works $[6,8]$ about stability of periodic traveling waves of systems of viscous conservation laws from the one-dimensional to the multi-dimensional setting.

M. Oh: Dept. Mathematics, University of Kansas, USA; moh@math.ku.edu K. Zumbrun: Dept. Mathematics, Indiana University, USA; kzumbrun@indiana.edu 
Consider a system of conservation laws

$$
u_{t}+\sum_{j} f^{j}(u)_{x_{j}}=\sum_{j, k}\left(B^{j k}(u) u_{x_{k}}\right)_{x_{j}}
$$

$u \in \mathcal{U}$ (open) $\subseteq \mathbb{R}^{n}, f^{j} \in \mathbb{R}^{n}, B^{j k} \in \mathbb{R}^{n \times n}, x \in \mathbb{R}^{d}, d \geq 2$, and a periodic traveling wave solution

$$
u=\bar{u}(x \cdot \nu-s t)
$$

of period $X$, satisfying the traveling wave ordinary differential equation

$$
\left(\sum_{j, k} \nu_{j} \nu_{k} B^{j k}(\bar{u}) \bar{u}^{\prime}\right)^{\prime}=\left(\sum_{j} \nu_{j} f^{j}(\bar{u})\right)^{\prime}-s \bar{u}^{\prime},
$$

with initial conditions

$$
\bar{u}(0)=\bar{u}(X)=: u_{0} .
$$

Integrating (2), we reduce to a first-order profile equation

$$
\sum_{j, k} \nu_{j} \nu_{k} B^{j k}(\bar{u}) \bar{u}^{\prime}=\sum_{j} \nu_{j} f^{j}(\bar{u})-s \bar{u}-q
$$

encoding the conservative structure of the equations, where $q$ is a constant of motion.

The one dimensional study was carried out by Oh \& Zumbrun [6] in the "quasi-hamiltonian" case that the traveling-wave equation possesses an integral of motion, and in the general case by Serre [8]. An important contribution of Serre was to point out a larger connection between the linearized dispersion relation (the function $\lambda(\xi)$ relating spectra to wave number of the linearized operator about the wave) near zero and the homogenized system obtained by slow modulation approximation, from which the various stability results of $[6,8]$ may then be deduced. The purpose of this paper is to extend to multiple dimensions this important observation of Serre, relating the linearized dispersion relation near zero to a multi-dimensional version of the homogenized system developed in [8]. As an immediate corollary, similarly as in $[6,8]$ in the onedimensional case, this yields as a necessary condition for multi-dimensional stability the hyperbolicity of the multi-dimensional homogenized system. In case of stability (so far not found), this relation is also the first step in the derivation of asymptotic behavior, as in [7] in the one-dimensional case; this we defer to a future investigation.

We here make only generic assumptions like those in [8], ensuring that the set of periodic traveling waves is a manifold of maximal dimension subject to the conservative properties of the equations (encoded in form (3)). Given $(a, s, \nu, q) \in \mathcal{U} \times \mathbb{R} \times S^{d-1} \times \mathbb{R}^{n}$, equation (3) admits a unique local solution 
$u(y ; a, s, \nu, q)$ such that $u(0 ; a, s, \nu, q)=a$. Denote by $X$ the period, by $\omega:=\frac{1}{X}$ the frequency and by $M$ and $F^{j}$ the averages over the period:

$$
M:=\frac{1}{X} \int_{0}^{X} u(y) d y, \quad F^{j}:=\frac{1}{X} \int_{0}^{X}\left(f^{j}(u)-\sum_{k=1}^{d} B^{j k}(u) \omega \nu_{k} \partial_{y} u\right) d y
$$

when $u$ is a periodic solution of (3). Since these quantities are translation invariant, we consider the set $P$ of periodic functions $u$ that are solutions of (3) for some triple $(s, \nu, q)$, and construct the quotient set $\mathcal{P}:=P / \mathcal{R}$ under the relation

$$
(u \mathcal{R} v) \Longleftrightarrow(\exists h \in \mathbb{R} ; v=u(\cdot-h)) .
$$

We thus have class functions:

$X=X(\dot{u}), \omega=\Omega(\dot{u}), s=S(\dot{u}), \nu=N(\dot{u}), q=Q(\dot{u}), M=M(\dot{u}), F^{j}=F^{j}(\dot{u})$,

where $\dot{u}$ is the equivalence class of translates of different periodic functions. Note that $\bar{u}$ is a nonconstant periodic solution. Without loss of generality, assume $S(\bar{u})=0$ and $N(\bar{u})=e_{1}$, so that (3) takes the form

$$
B^{11}(\bar{u}) \bar{u}^{\prime}=f^{1}(\bar{u})-\bar{q}
$$

for $\bar{q}=Q(\bar{u})$. Letting $\bar{X}=X(\bar{u})$ and $\bar{a}=\bar{u}(0)=u_{0}$, the map $(y, a, s, \nu, q) \mapsto$ $u(y ; a, s, \nu, q)-a$ is smooth and well-defined in a neighborhood of $\left(\bar{X} ; \bar{a}, 0, e_{1}, \bar{q}\right)$, and it vanishes at this special point. Here and elsewhere, $e_{j}$ denotes the $j$ th standard Euclidean basis element. We assume:

(H0) $f^{j}, B^{j k} \in C^{2}$.

(H1) $\operatorname{Re} \sigma\left(\sum_{j k} \nu_{j} \nu_{k} B^{j k}\right) \geq \theta>0$.

(H2) The map $H: \mathbb{R} \times \mathcal{U} \times \mathbb{R} \times S^{d-1} \times \mathbb{R}^{n} \rightarrow \mathbb{R}^{n}$ taking $(X ; a, s, \nu, q) \mapsto$ $u(X ; a, s, \nu, q)-a$ is a submersion at point $\left(\bar{X} ; \bar{a}, 0, e_{1}, \bar{q}\right)$.

As a consequence of (H0), (H2), there is a smooth $n+d$ dimensional manifold $\mathcal{P}$ of periodic solutions $\dot{u}$ in the vicinity of $\bar{u}$, where $d$ is the spatial dimension. On this set, one may obtain, rescaling by $(x, t) \rightarrow(\epsilon x, \epsilon t)$ and carrying out a formal WKB expansion as $\epsilon \rightarrow 0$ a closed system of $n+d$ averaged, or homogenized, equations

$$
\begin{aligned}
& \partial_{t} M(\dot{u})+\sum_{j} \partial_{x_{j}}\left(F^{j}(\dot{u})\right)=0 \\
& \partial_{t}(\Omega N(\dot{u}))+\nabla_{x}(\Omega S(\dot{u}))=0
\end{aligned}
$$

in the $(n+d)$-dimensional unknown $\dot{u}$, expected to correspond to large timespace behavior. For details, see Section 4. The problem of stability of $\bar{u}$ may heuristically be expected to be related to the linearized equations of (4) about 
the constant solution $\dot{u}(x, t) \equiv u^{0}, u^{0} \sim \bar{u}$, provided that the WKB expansion is justifiable by stability considerations. This leads to the homogeneous degree $n+d$ linearized dispersion relation

$$
\hat{\Delta}(\xi, \lambda):=\operatorname{det}\left(\lambda \frac{\partial(M, \Omega N)}{\partial \dot{u}}(\dot{\bar{u}})+\sum_{j} i \xi_{j} \frac{\partial\left(F^{j}, S \Omega e_{j}\right)}{\partial \dot{u}}(\dot{\bar{u}})\right)=0 .
$$

On the other hand, one may also pursue the direct course of linearizing PDE (1) about the stationary solution $\bar{u}$ and studying the spectrum of the associated linearized operator $L$. Taking the Fourier transform in constant directions $x_{j}, j \neq 1$, and following the general construction of $[1,6]$ we obtain an Evans function $D(\xi, \lambda), \xi \in \mathbb{R}^{d}, \lambda \in \mathbb{C}$, of which the zero set $(\xi, \lambda(\xi))$ determines the linearized dispersion relation for $(1)$, with $\lambda(\xi)$ running over the spectrum of $L$ as $\xi$ runs over $\mathbb{R}^{d}$. For details, see Section 2. In particular, the low-frequency expansion of $\lambda(\xi)$ near $(\xi, \lambda)=(0,0)$ may be expected to determine long-time asymptotic behavior, provided that the spectrum away from $\lambda=0$ has a strictly negative real part, and this in turn may be expected to derive from the lowest order terms of the Taylor expansion of $D$. A tedious, but fairly straightforward calculation following $[6,8]$ shows that

$$
D(\xi, \lambda)=\Delta_{1}(\xi, \lambda)+\mathcal{O}\left(|\xi, \lambda|^{n+2}\right)
$$

where $\Delta_{1}$ is a homogeneous degree $n+1$ polynomial expressed as the determinant of a rather complicated $2 n \times 2 n$ matrix in $(\xi, \lambda)$ : in particular, not in the simple form det $\lambda N_{0}+\sum_{j} i \xi_{j} N_{j}$ of a first-order hyperbolic $(n+1) \times(n+1)$ dispersion relation, or an obvious tensor product thereof.

Our main result is the following theorem relating these two expansions, generalizing the result of [8] in the one-dimensional case. Define

$$
\Delta(\xi, \lambda):=\lambda^{1-d} \hat{\Delta}(\xi, \lambda),
$$

where $\hat{\Delta}$ is defined as in (5).

Theorem 1.1. Under the assumptions (H0)-(H2), $\Delta_{1}=\Gamma_{0} \Delta$, i.e.,

$$
D(\xi, \lambda)=\Gamma_{0} \Delta(\xi, \lambda)+\mathcal{O}\left(|\xi, \lambda|^{n+2}\right)
$$

$\Gamma_{0} \neq 0$ constant, for $|\xi, \lambda|$ sufficiently small.

That is, up to an additional factor of $\lambda^{d-1}$, the dispersion relation (5) for the averaged system (4) indeed describes the low-frequency limit of the exact linearized dispersion relation

$$
D(\xi, \lambda)=0
$$

The discrepancy $\lambda^{d-1}$ is an interesting and at first glance puzzling new phenomenon in the multi-dimensional case. However, it is easily explained by a 
closer look at the formal approximation procedure described in Section 4. For, in the derivation of (4), it was assumed that $\Omega N$ represent the gradient $\nabla_{x} \phi$ of a certain phase function $\phi(x, t)$. In one dimension, this is no restriction, since we may always take $\phi(x, t):=\int_{0}^{x} \omega(z) d z$. However, in multidimensions, it imposes the additional constraint

$$
\operatorname{curl}(\Omega N) \equiv 0
$$

which properly should be adjoined to the averaged system.

Taking the curl of the second equation of (4), we obtain the simple equation

$$
\partial_{t} \operatorname{curl}(\Omega N)=0
$$

revealing at once that constraint (8) is compatible with the time-evolution of the system, and that the unconstrained system possesses $(d-1)$ spurious zero characteristices $\lambda(\xi) \equiv 0$, corresponding to the $(d-1)$ Fourier modes in the range of the curl operator $\hat{f} \rightarrow \xi$ curl $\hat{f}$, lying in $(\xi /|\xi|)^{\perp}$. Thus, $\Delta(\xi, \lambda)=$ $\lambda^{1-d} \hat{\Delta}(\xi, \lambda)=0$ is exactly the linearized dispersion relation for the constrained averaged system (4), (8) relevant to time-asymptotic behavior.

Theorem 1.1 may be regarded as partial justification of the WKB expansion. Roughly speaking, it states that if perturbed periodic waves exhibit coherent behavior near the unperturbed wave $\bar{u}$, then this behavior is well-described by the constrained averaged equations (4), (8). In one dimension, additional results of [7] case give rigorous sense to this statement in the form of detailed pointwise linear bounds under the assumption of spectral stability of the linearized operator about the wave. To establish a comparable long time result on behavior in multidimensions would be a very interesting direction for future investigation. Moreover, the new description of the solution given in [8] by modulation expansion might give a sufficiently good nonlinear Ansatz to carry out a complete nonlinear analysis, which was not done even in the one-dimensional case. Thus, it would be interesting to revisit also the one-dimensional setting of [7] from this new perspective, in particular, to resolve certain puzzling issues in the general, non-quasi-Hamiltonian case.

An equally interesting direction for future investigation would be to rigorously validate the WKB expansion of Section 4 for the closely related small viscosity problem

$$
u_{t}+\sum_{j} f^{j}(u)_{x_{j}}=\epsilon \sum_{j, k}\left(B^{j k}(u) u_{x_{k}}\right)_{x_{j}}
$$

$\epsilon \rightarrow 0$, similarly as in $[3,4]$ for the viscous shock case. We note in this regard that a key ingredient in the [3, 4] analysis, the conjugation lemma of [5] asserting the existence of a coordinate change converting an asymptotically constantcoefficient resolvent ODE to constant-coefficient form, has a straightforward 
analog in the standard Floquet construction converting periodic- to constantcoefficient $O D E$; indeed, this construction is more natural in the periodic case. Thus, the whole Kreiss symmetrizer construction of $[3,4]$ may be brought to bear also in the periodic case. Indeed, the situation is simpler since no shock front appears; as remarked in [7], there is a much closer analogy to the constantcoefficient case. Assuming successful validation of the modulation equations, there is also the interesting question of secondary modulation, i.e., what happens after shock formation time for the averaged, hyperbolic equations?

We point out further, as observed by Serre [8] in the one-dimensional case, that the result of Theorem 1.1 is completely analogous to the corresponding relation established in [12] for the linearized dispersion relation associated with a perturbed viscous traveling front $u=\bar{u}(x \cdot \nu-s t), \lim _{z \rightarrow \pm \infty}=u_{ \pm}$, in which the WKB expansion corresponds to matched asymptotics joining an outer, hyperbolic solution and an inner, viscous profile. See [11, Section 1.3] for a formal derivation in the shock case, starting with the same rescaling $(x, t) \rightarrow(\epsilon x, \epsilon t)$; see also the related discussion of long-time vs. small-viscosity problems in [2]. Rigorous verification may be found in $[2,11]$.

As an immediate consequence of Theorem 1.1, we obtain the following two corollaries, yielding a necessary condition for low-frequency multi-dimensional spectral stability strengthening the one-dimensional version obtained in $[6,8]$.

Corollary 1.2. Assuming $(\mathrm{H} 0)-(\mathrm{H} 2)$ and the nondegeneracy condition

$$
\operatorname{det}\left(\frac{\partial(M, \Omega N)}{\partial \dot{u}}(\dot{\bar{u}})\right) \neq 0
$$

then for $\lambda, \xi$ sufficiently small, the zero-set of $D(\cdot, \cdot)$, corresponding to spectra of $L$, consists of $n+1$ characteristic surfaces:

$$
\lambda_{j}(\xi)=-i a_{j}(\xi)+o(\xi), \quad j=1, \ldots, n+1,
$$

where $a_{j}(\xi)$ denote the eigenvalues of

$$
\mathcal{A}:=\sum_{j} \xi_{j} \frac{\partial\left(F^{j}, S \Omega e_{j}\right)}{\partial(M, \Omega N)},
$$

excluding $(d-1)$ identically zero eigenvalues associated with modes not satisfying constraint (8).

Proof. Similarly as in the proof of the analogous Lemma 7.5 in [12] in the shock wave case, assuming (9), we may easily deduce (10) from (7) using Rouché's Theorem. Defining

$$
\mathcal{D}^{\rho, \hat{\xi}}(\hat{\lambda}):=\rho^{-(n+1)} D(\rho \hat{\xi}, \rho \hat{\lambda})
$$


for $(\rho, \hat{\xi}, \hat{\lambda}) \in \mathbb{R} \times S^{d-1} \times \mathbb{C}$, we obtain a $d$-parameter family of analytic maps, converging as $\rho \rightarrow 0$ to $\mathcal{D}^{0, \hat{\xi}}=\Delta(\hat{\xi}, \cdot)$. Under assumption $(9), \mathcal{D}^{0, \hat{\xi}}=\Delta(\hat{\xi}, \cdot) \sim$ $\hat{\lambda}^{n+1}$ as $|\lambda| \rightarrow \infty$, hence, for $\rho$ sufficiently small, $\mathcal{D}^{\rho, \hat{\xi}}$ has $n+1$ continuously varying $\operatorname{roots} \lambda=\hat{a}_{j}(\hat{\xi}, \rho)$. Defining $a_{j}(\xi):=|\xi| \hat{a}_{j}(\xi /|\xi|,|\xi|)$, we obtain the result.

Remark 1.3. Evidently, $a_{j}(\xi)$ are smooth in $|\xi|$ for fixed $\hat{\xi}$, but in general have a conical singularity at $\xi=0$ when considered as a function of $\xi$, i.e., $\partial a_{j} / \partial \xi$ is discontinuous at $\xi=0$.

Corollary 1.4. Assuming (H0)-(H2) and the nondegeneracy condition (9), a necessary condition for low-frequency spectral stability of $\bar{u}$, defined as $\operatorname{Re} \lambda \leq 0$ for $D(\xi, \lambda)=0, \xi \in \mathbb{R}^{d}$, and $|\xi, \lambda|$ sufficiently small, is that the averaged system (4) be "weakly hyperbolic" in the sense that it possesses a full set of real characteristics $\hat{\lambda}_{j}(\xi)$ for each $\xi \in \mathbb{R}^{d}$, i.e., the eigenvalues of

$$
\mathcal{A}=\sum_{j} \xi_{j} \frac{\partial\left(F^{j}, S \Omega e_{j}\right)}{\partial(M, \Omega N)}
$$

are real.

Remark 1.5. Condition (9), or equivalently $(\partial / \partial \lambda)^{n+1} D(0,0) \neq 0$, is a necessary condition for one-dimensional linearized stability [7], while hyperbolicity is necessary for stability of the homogenized system linearized about a constant state. Thus, Corollaries 1.2 and 1.4 are analogous to results of [12] in the shock wave case, stating that, given one-dimensional stability, stability of the inviscid equations linearized about an ideal shock is necessary for multi-dimensional stability of a viscous shock wave.

Finally, we mention that, though the averaged system may in some cases be hyperbolic [6], so far, only unstable periodic traveling-wave solutions have been found for viscous conservation laws. However, essentially only the single, $2 \times 2$ model of van der Waals gas dynamics with viscosity - capillarity in one dimension has so far been considered in detail $[6,8,9]$ and we see no obvious reason why a stable wave should not exist for other models. It would be extremely interesting to either find such an example, with the associated rich behavior described by the modulation equations, or show that it can in no case exist. As suggested by Serre [10], a useful starting point might be to consider whether the averaged system (4) might ever possess an entropy.

Plan of the paper. In Section 2, we recall the basic Evans function construction of [1]. In Section 3, we carry out the expansion (6), and in Section 4, the multi-dimensional WKB expansion (4). Finally, in Section 5, we carry out the proof of Theorem 1.1 by a calculation similar to the one used by Serre [8] to treat the one-dimensional case. 


\section{Preliminaries}

Without loss of generality taking $S(\bar{u})=0, N(\bar{u})=e_{1}, \bar{u}=\bar{u}\left(x_{1}\right)$ represents a stationary solution. Linearizing (1) about $\bar{u}(\cdot)$, we obtain

$$
v_{t}=L v:=\sum\left(B^{j k} v_{x_{k}}\right)_{x_{j}}-\sum\left(A^{j} v\right)_{x_{j}}
$$

where the coefficients

$$
B^{j k}:=B^{j k}(\bar{u}), \quad A^{j} v:=D f^{j}(\bar{u}) v-\left(D B^{j 1}(\bar{u}) v\right) \bar{u}_{x_{1}}
$$

are now periodic functions of $x_{1}$.

Taking the Fourier transform in the transverse coordinate $\tilde{x}=\left(x_{2}, \ldots, x_{d}\right)$, we obtain

$$
\begin{aligned}
\hat{v}_{t}=L_{\tilde{\xi}} \hat{v}= & \left(B^{11} \hat{v}_{x_{1}}\right)_{x_{1}}-\left(A^{1} \hat{v}\right)_{x_{1}}+i\left(\sum_{j \neq 1} B^{j 1} \xi_{j}\right) \hat{v}_{x_{1}} \\
& +i\left(\sum_{k \neq 1} B^{1 k} \xi_{k} \hat{v}\right)_{x_{1}}-i \sum_{j \neq 1} A^{j} \xi_{j} \hat{v}-\sum_{j \neq 1, k \neq 1} B^{j k} \xi_{k} \xi_{j} \hat{v} .
\end{aligned}
$$

The Laplace transform in time $t$ leads us to study the family of eigenvalue equations

$$
\begin{aligned}
0=\left(L_{\tilde{\xi}}-\lambda\right) w= & \left(B^{11} w^{\prime}\right)^{\prime}-\left(A^{1} w\right)^{\prime}+i \sum_{j \neq 1} B^{j 1} \xi_{j} w^{\prime}+i\left(\sum_{k \neq 1} B^{1 k} \xi_{k} w\right)^{\prime} \\
& -i \sum_{j \neq 1} A^{j} \xi_{j} w-\sum_{j \neq 1, k \neq 1} B^{j k} \xi_{k} \xi_{j} w-\lambda w
\end{aligned}
$$

associated with operators $L_{\tilde{\xi}}$ and frequency $\lambda \in \mathbb{C}$, where " denotes $\partial / \partial x_{1}$. Clearly, a necessary condition for stability of (1) is that equations (13) have no $L^{2}$ solutions $w$ for $\tilde{\xi} \in \mathbb{R}^{d-1}$ and $\operatorname{Re} \lambda>0$. For solutions of (13) correspond to normal modes $\hat{v}(x, t)=\mathrm{e}^{\lambda t} \mathrm{e}^{i \tilde{\xi} \cdot \tilde{x}} w\left(x_{1}\right)$ of $(11)$.

The difficulty of our problem is due to accumulation at the origin of the essential spectrum of the linearized operator $L$ about the wave as in the one dimensional case. Multidimensional stability concerns the behavior of the perturbation of the top eigenvalue, $\lambda=0$ under small perturbations in $\tilde{\xi}$. To study this stability, we use Floquet's theory and an Evans function [1] which not only depends on $\lambda$ but also on $\xi_{1}$ which corresponds to the phase shift and $\tilde{\xi}$. To define the Evans function, we choose a basis $\left\{w^{1}\left(x_{1}, \tilde{\xi}, \lambda\right), \ldots, w^{2 n}\left(x_{1}, \tilde{\xi}, \lambda\right)\right\}$ of the kernel of $L_{\tilde{\xi}}-\lambda$, which is analytic in $(\tilde{\xi}, \lambda)$ and is real when $\lambda$ is real, for details see $[6,8]$. Now we can define the Evans function by

$$
D\left(\lambda, \xi_{1}, \tilde{\xi}\right):=\left|\begin{array}{c}
w^{l}(X, \tilde{\xi}, \lambda)-\mathrm{e}^{i X \xi_{1}} w^{l}(0, \tilde{\xi}, \lambda) \\
\left(w^{l}\right)^{\prime}(X, \tilde{\xi}, \lambda)-\mathrm{e}^{i X \xi_{1}}\left(w^{l}\right)^{\prime}(0, \tilde{\xi}, \lambda)
\end{array}\right|_{1 \leq l \leq 2 n}
$$


where $\xi_{1} \in \mathbb{R}$. Note that $X \xi_{1}$ is exactly $\theta$ in [8]. We remark that $D$ is analytic everywhere, with associated analytic eigenfunction $w^{l}$ for $1 \leq l \leq 2 n$. A point $\lambda$ is in the spectrum of $L_{\tilde{\xi}}$ if and only if $D(\lambda, \xi)=0$ with $\xi=\left(\xi_{1}, \tilde{\xi}\right)$.

Example 2.1. In the constant-coefficient case

$$
\begin{gathered}
B^{11} w^{\prime \prime}-A^{1} w^{\prime}+i \sum_{j \neq 1} B^{j 1} \xi_{j} w^{\prime}+i \sum_{k \neq 1} B^{1 k} \xi_{k} w^{\prime} \\
-i \sum_{j \neq 1} A^{j} \xi_{j} w-\sum_{j \neq 1, k \neq 1} B^{j k} \xi_{k} \xi_{j} w-\lambda w=0,
\end{gathered}
$$

an elementary computation yields

$$
D(\lambda, \xi)=\prod_{l=1}^{2 n}\left(\mathrm{e}^{\mu_{l}(\lambda, \tilde{\xi}) X}-\mathrm{e}^{i \xi_{1} x}\right)
$$

where $\mu_{l}, l=1, \ldots, 2 n$, denote the roots of the characteristic equation

$$
\begin{aligned}
& \left(\mu^{2} B^{11}+\mu\left(-A^{1}+i \sum_{j \neq 1} B^{j 1} \xi_{j}+i \sum_{k \neq 1} B^{1 k} \xi_{k}\right)\right. \\
& \left.-\left(i \sum_{j \neq 1} A^{j} \xi_{j}+\sum_{j \neq 1, k \neq 1} B^{j k} \xi_{k} \xi_{j}+\lambda I\right)\right) \bar{w}=0,
\end{aligned}
$$

where $w=\mathrm{e}^{\mu x_{1}} \bar{w}$. The zero set of $D$ consists of all $\lambda$ and $\xi_{1}$ such that

$$
\mu_{l}(\lambda, \tilde{\xi})=i \xi_{1}(\bmod 2 \pi i / X)
$$

for some $l$. Setting $\mu=i \xi_{1}$ in (15), we obtain the dispersion relation

$$
\operatorname{det}\left(-B^{\xi}-i A^{\xi}-\lambda I\right)=0
$$

where $A^{\xi}=\sum_{j} A^{j} \xi_{j}$ and $B^{\xi}=\sum_{j, k} B^{j k} \xi_{k} \xi_{j}$.

Remark 2.2. If $(\lambda, \tilde{\xi})=(0,0)$, then (15) reduces to $\mu\left(\left(B^{11}\right)^{-1} A^{1}-\mu\right) \bar{w}=0$ giving $n$ nonzero roots $\mu=s_{j}, \quad \bar{w}=t_{j}$, where $s_{j}, t_{j}$ are eigenvalues and eigenvectors for the matrix $\left(B^{11}\right)^{-1} A^{1}$, and an $n$-fold root $\mu=0$. Thus, $D(0,0)=0$ in the above example. We shall see later that this holds also in the general variable-coefficient case.

Remark 2.3. In the constant coefficient case, (16) yields expansions

$$
\lambda_{j}(\xi)=0-i a_{j}(\xi)+o(|\xi|), \quad j=1, \ldots, n,
$$

for the $n$ roots bifurcating from $\lambda(0)=0$, where $a_{j}$ denote the eigenvalues of $A^{\xi}$. Thus we obtain the necessary stability condition of hyperbolicity, $\sigma\left(A^{\xi}\right)$ real. 


\section{Evans function calculations}

Motivated by the example, we now find linearized dispersion relations for the variable-coefficient Evans function in the low-frequency limit. From now on, coordinatize $\nu$ in the vicinity of $e_{1}$ by

$$
\nu=: \frac{\left(1, \delta^{2}, \ldots, \delta^{d}\right)}{\sqrt{1+|\delta|^{2}}},
$$

$\delta=\left(\delta^{2}, \ldots, \delta^{d}\right) \in \mathbb{R}^{d-1}$. Note that differentiation of $(17)$ yields $\partial \nu=(0, \delta)$.

Lemma 3.1 ([8]). Assumption (H2) is equivalent to

$$
\mathbb{R}^{n}=\operatorname{span}\left\{\left[\frac{\partial u}{\partial s}\right],\left[\frac{\partial u}{\partial \delta}\right],\left[w^{2}\right], \ldots,\left[w^{2 n}\right], \bar{u}^{\prime}(0)\right\} .
$$

Proof. Immediate, using $\left[w_{1}\right]=0$; see [8] for the one-dimensional case.

3.1. Variational relations. If $\tilde{\xi}=0$, equation (13) becomes

$$
\left(L_{0}-\lambda\right) w=\left(B^{11} w^{\prime}\right)^{\prime}-\left(A^{1} w\right)^{\prime}-\lambda w=0,
$$

which is associated with the one dimensional stability problem studied in $[6,8]$. Recall that $\bar{u}$ is $\bar{X}$-periodic in $x_{1}$ and the functions $w^{1}\left(x_{1}, \tilde{\xi}, \lambda\right), \ldots, w^{2 n}\left(x_{1}, \tilde{\xi}, \lambda\right)$ are in the basis of the kernel of $L_{\tilde{\xi}}-\lambda$. Following [6], we normalize

$$
\begin{aligned}
w^{j}(0, \tilde{\xi}, \lambda) & =e_{j}, & \left(w^{j}\right)^{\prime}(0, \tilde{\xi}, \lambda) & =\left(B^{11}\right)^{-1} A^{1} e_{j} \\
w^{n+j}(0, \tilde{\xi}, \lambda) & =0, & \left(w^{n+j}\right)^{\prime}(0, \tilde{\xi}, \lambda) & =-\left(B^{11}\right)^{-1} e_{j}
\end{aligned}
$$

for $j=1, \ldots, n$ and all $(\tilde{\xi}, \lambda)$, giving in particular

$$
\hat{L} w^{j}(0, \tilde{\xi}, \lambda)=0, \quad \hat{L} w^{n+j}(0, \tilde{\xi}, \lambda)=e_{j} \quad \text { for } j=1, \ldots, n .
$$

Plug the Taylor expansion of $w\left(x_{1}, \tilde{\xi}, \lambda\right)$ at the origin of $(\lambda, \tilde{\xi})$

$$
\begin{aligned}
& w^{l}(\cdot, \tilde{\xi}, \lambda) \\
& =w^{l}(\cdot, 0,0)+\lambda w_{\lambda}^{l}(\cdot, 0,0)+\sum_{j \neq 1} w_{\xi_{j}}^{l}(\cdot, 0,0) \xi_{j} \\
& \quad+\frac{1}{2}\left(\lambda^{2} w_{\lambda \lambda}^{l}(\cdot, 0,0)+2 \lambda \sum_{j \neq 1} w_{\lambda \xi_{j}}^{l}(\cdot, 0,0) \xi_{j}+\sum_{j \neq 1, k \neq 1} w_{\xi_{j} \xi_{k}}^{l}(\cdot, 0,0) \xi_{k} \xi_{j}\right)+\ldots
\end{aligned}
$$

into (13) to find the identities

$$
\left(\hat{L} w^{l}\right)^{\prime}=0, \quad\left(\hat{L} w_{\lambda}^{l}\right)^{\prime}=w^{l}, \quad\left(\hat{L} w_{\lambda \lambda}^{l}\right)^{\prime}=2 w_{\lambda}^{l}
$$


and

$$
\begin{aligned}
\left(\hat{L} w_{\lambda \xi_{j}}^{l}\right)^{\prime}= & i A^{j} w_{\lambda}^{l}-i B^{j 1}\left(w_{\lambda}^{l}\right)^{\prime}-i\left(B^{1 j} w_{\lambda}^{l}\right)^{\prime}+w_{\xi_{j}}^{l} \\
\left(\hat{L} w_{\xi_{j} \xi_{k}}^{l}\right)^{\prime}= & i A^{j} w_{\xi_{k}}^{l}+i A^{k} w_{\xi_{j}}^{l}-i B^{j 1}\left(w_{\xi_{k}}^{l}\right)^{\prime}-i\left(B^{1 j} w_{\xi_{k}}^{l}\right)^{\prime}-i B^{k 1}\left(w_{\xi_{j}}^{l}\right)^{\prime} \\
& -i\left(B^{1 k} w_{\xi_{j}}^{l}\right)^{\prime}+2 B^{j k} w^{l},
\end{aligned}
$$

where $\hat{L} w=B^{11} w^{\prime}-A^{1} w$, and also

$$
\left(\hat{L} w_{\xi_{j}}^{l}\right)^{\prime}=i\left(A^{j} w^{l}-B^{j 1}\left(w^{l}\right)^{\prime}-\left(B^{1 j} w^{l}\right)^{\prime}\right), \quad j \neq 1
$$

and

$$
\left(\hat{L} w_{\xi_{j}}^{1}\right)^{\prime}=i\left(f^{j}(\bar{u})-B^{j 1}(\bar{u}) \bar{u}^{\prime}-B^{1 j}(\bar{u}) \bar{u}^{\prime}\right)^{\prime}, \quad j \neq 1
$$

by using the definition of $A^{j}$ in (12). In the Laplacian case $B^{j k}=\delta_{k}^{j}$, the latter identity simplifies to

$$
\left(\hat{L} w_{\xi_{j}}^{1}\right)^{\prime}=i f^{j}(\bar{u})^{\prime} .
$$

Note that the functions $w^{1}\left(x_{1}, 0,0\right), \ldots, w^{2 n}\left(x_{1}, 0,0\right)$ are in the basis of the kernel of $L_{0}$. We omit $(\cdot, 0,0)$ hereabove and denote $[w]=w(X)-w(0)$. We also have $w^{1}=\bar{u}^{\prime}, \hat{L} w^{1}=0,\left[w^{1}\right]=0, \int_{0}^{\bar{X}} w^{1} d x_{1}=0$, and moreover,

$$
\begin{array}{ll}
{\left[\hat{L} w^{l}\right]=0,} & {\left[\hat{L} w_{\lambda}^{l}\right]=\int_{0}^{\bar{X}} w^{l} d x_{1}} \\
{\left[\hat{L} w_{\lambda}^{1}\right]=0,} & {\left[\hat{L} w_{\xi_{j}}^{1}\right]=0}
\end{array}
$$

and

$$
\begin{aligned}
{\left[\hat{L} w_{\lambda \lambda}^{1}\right]=} & 2 \int_{0}^{\bar{X}} w_{\lambda}^{1} d x_{1} \\
{\left[\hat{L} w_{\lambda \xi_{j}}^{1}\right]=} & \int_{0}^{\bar{X}}\left(i A^{j} w_{\lambda}^{1}-i B^{j 1}\left(w_{\lambda}^{l}\right)^{\prime}-i\left(B^{1 j} w_{\lambda}^{1}\right)^{\prime}+w_{\xi_{j}}^{1}\right) d x_{1} \\
{\left[\hat{L} w_{\xi_{j} \xi_{k}}^{1}\right]=} & \int_{0}^{\bar{X}}\left(i A^{j} w_{\xi_{k}}^{1}+i A^{k} w_{\xi_{j}}^{1}-i B^{j 1}\left(w_{\xi_{k}}^{1}\right)^{\prime}-i\left(B^{1 j} w_{\xi_{k}}^{1}\right)^{\prime}\right. \\
& \left.-i B^{k 1}\left(w_{\xi_{j}}^{1}\right)^{\prime}-i\left(B^{1 k} w_{\xi_{j}}^{1}\right)^{\prime}+2 B^{j k} w^{1}\right) d x_{1} .
\end{aligned}
$$

In the Laplacian case $B^{j k}=\delta_{k}^{j}$, the last two identities simplify considerably to

$$
\begin{aligned}
{\left[\hat{L} w_{\lambda \xi_{j}}^{1}\right] } & =\int_{0}^{\bar{X}}\left(i A^{j} w_{\lambda}^{1}+w_{\xi_{j}}^{1}\right) d x_{1} \\
{\left[\hat{L} w_{\xi_{j} \xi_{k}}^{1}\right] } & =\int_{0}^{\bar{X}}\left(i A^{j} w_{\xi_{k}}^{1}+i A^{k} w_{\xi_{j}}^{1}\right) d x_{1} .
\end{aligned}
$$


3.2. Connection to traveling-wave variations. From (18), we find easily that

$$
w^{j}(\cdot, 0,0)=\left.\frac{\partial u}{\partial a_{j}}\right|_{\bar{u}}, \quad w^{n+j}(\cdot, 0,0)=\left.\frac{\partial u}{\partial q_{j}}\right|_{\bar{u}} \quad \text { for } j=1, \ldots, n .
$$

For example, taking the variation of traveling wave equation (3) with respect to $q_{j}$, we find that $z=\frac{\partial u}{\partial q_{j}}$ satisfies

$$
B^{11} z^{\prime}-A^{1} z=-e_{j}
$$

with $z(0)=0$, so that $L_{0} z=0$ and $z^{\prime}(0)=-\left(B^{11}\right)^{-1} e_{j}$ as claimed.

Further (see $[6,8])$

$$
w_{\lambda}^{1}=-\frac{\partial u}{\partial s}+\sum_{n+1}^{2 n} \alpha^{l} w^{l}
$$

for $\alpha \in \mathbb{R}^{2 n}$, since $L_{0}\left(-\frac{\partial u}{\partial s}\right)=L_{0} w_{\lambda}^{1}(\cdot, 0,0)=\bar{u}^{\prime}$ and $w_{\lambda}^{1}(0,0,0)=\left(\frac{\partial u}{\partial s}\right)(0)=0$, and, similarly, for $j \neq 1$, using $L_{0} w_{\xi_{j}}^{1}(\cdot, 0,0)=L_{0}\left(i \frac{\partial u}{\partial \delta^{j}}\right)(\cdot, 0,0)=i f^{j}(\bar{u})^{\prime}$ and $w_{\xi_{j}}^{1}(0,0,0)=\frac{\partial u}{\partial \delta^{j}}(0)=0$,

$$
w_{\xi_{j}}^{1}(\cdot, 0,0)=i \frac{\partial u}{\partial \delta^{j}}+\sum_{n+1}^{2 n} \beta_{j}^{l} w^{l}
$$

Alternatively,

$$
\tilde{w}_{\lambda}^{1}(\cdot, 0,0)=-\frac{\partial u}{\partial s}, \quad \tilde{w}_{\xi_{j}}^{1}(\cdot, 0,0)=i \frac{\partial u}{\partial \delta^{j}}
$$

for

$$
\tilde{w}^{1}:=w^{1}-\lambda \sum_{n+1}^{2 n} \alpha^{l} w^{l}-\sum_{\ell=n+1}^{2 n} \sum_{j} \xi_{j} \beta_{j}^{l} w^{l},
$$

with $\tilde{w}^{1}(0,0)$ still equal to $\bar{u}^{\prime}$. We hereafter substitute $\tilde{w}^{1}$ for $w^{1}$ everywhere it appears, as we are free to do. (Recall, $w^{\ell}$ can be an arbitrary basis of the kernel of $L$.)

3.3. Reduction of the leading part. We rewrite the Evans function (14) as

$$
D\left(\lambda, \xi_{1}, \tilde{\xi}\right):=\left|\begin{array}{c}
{\left[w^{l}(\tilde{\xi}, \lambda)\right]+\left(1-\mathrm{e}^{i \bar{X} \xi_{1}}\right) w^{l}(0, \tilde{\xi}, \lambda)} \\
{\left[\left(w^{l}\right)^{\prime}(\tilde{\xi}, \lambda)\right]+\left(1-\mathrm{e}^{i \bar{X} \xi_{1}}\right)\left(w^{l}\right)^{\prime}(0, \tilde{\xi}, \lambda)}
\end{array}\right|_{1 \leq l \leq 2 n}
$$

and then multiply the second row in (20) by $B^{11}$ and then subtract $A^{1}$ times the first one

$$
\left(\operatorname{det} B^{11}\right) D\left(\lambda, \xi_{1}, \tilde{\xi}\right):=\left|\begin{array}{c}
{\left[w^{l}(\tilde{\xi}, \lambda)\right]+\left(1-\mathrm{e}^{i \bar{X} \xi_{1}}\right) w^{l}(0, \tilde{\xi}, \lambda)} \\
{\left[\hat{L} w^{l}(\tilde{\xi}, \lambda)\right]+\left(1-\mathrm{e}^{i \bar{X} \xi_{1}}\right)\left(\hat{L} w^{l}\right)(0, \tilde{\xi}, \lambda)}
\end{array}\right|_{1 \leq l \leq 2 n} .
$$


At this point, we restrict for readability to the Laplacian case $B^{j k}=\delta_{k}^{j}$. The general case goes similarly. Then the Evans function $D\left(\lambda, \xi_{1}, 0\right)$ becomes

$$
\Gamma_{0} \operatorname{det}\left(\begin{array}{ccc}
c(\xi, \lambda) & {\left[w^{2}(0,0)\right]} & \ldots \\
C(\xi, \lambda) & C^{2}(\xi, \lambda) & \ldots
\end{array}\right)+\mathcal{O}\left(|\lambda|^{n+2}+\left|\xi_{1}\right|^{n+2}\right)
$$

with a nonzero number $\Gamma_{0}$ (for details, see $[6,8]$ ), where

$$
\begin{aligned}
c(\xi, \lambda) & =\lambda\left[w_{\lambda}^{1}\right]+\sum_{j \neq 1} \xi_{j}\left[w_{\xi_{j}}^{1}\right]-i \bar{X} \xi_{1} \bar{u}^{\prime}(0) \\
& =-\lambda\left[\frac{\partial u}{\partial s}\right]+\sum_{j \neq 1} i \xi_{j}\left[\frac{\partial u}{\partial \delta_{j}}\right]-i \bar{X} \xi_{1} \bar{u}^{\prime}(0)
\end{aligned}
$$

is a homogeneous degree one polynomial,

$$
\begin{aligned}
C(\xi, \lambda):= & \frac{1}{2} \lambda^{2}\left[\hat{L} w_{\lambda \lambda}^{1}\right]+\lambda \sum_{j \neq 1} \xi_{j}\left[\hat{L} w_{\lambda \xi_{j}}^{1}\right]+\frac{1}{2} \sum_{j, k \neq 1} \xi_{j} \xi_{k}\left[\hat{L} w_{\xi_{j} \xi_{k}}^{1}\right] \\
& -i \bar{X} \xi_{1} \lambda\left(\hat{L} w_{\lambda}^{1}\right)(0)-i \bar{X} \xi_{1} \sum_{j \neq 1} \xi_{j}\left(\hat{L} w_{\xi_{j}}^{1}\right)(0) \\
= & -\lambda^{2} \int_{0}^{\bar{X}} \frac{\partial u}{\partial s} d x_{1}+i \lambda \sum_{j \neq 1} \xi_{j} \int_{0}^{\bar{X}}\left(A^{j}\left(-\frac{\partial u}{\partial s}\right)+\frac{\partial u}{\partial \delta^{j}}\right) d x_{1} \\
& -\sum_{j, k \neq 1} \xi_{j} \xi_{k} \int_{0}^{\bar{X}} A^{j}\left(\frac{\partial u}{\partial \delta^{k}}\right) d x_{1} \\
& +i \bar{X} \xi_{1} \lambda\left(\hat{L} \frac{\partial u}{\partial s}\right)(0)+\bar{X} \xi_{1} \sum_{j \neq 1} \xi_{j}\left(\hat{L} \frac{\partial u}{\partial \delta^{j}}\right)(0) \\
= & -\lambda^{2} \int_{0}^{\bar{X}}\left(\frac{\partial u}{\partial s}\right) d x_{1}+i \lambda \sum_{j \neq 1} \xi_{j}\left(-\frac{\partial}{\partial s} \int_{0}^{\bar{X}} f^{j}(u) d x_{1}+\frac{\partial}{\partial \delta^{j}} \int_{0}^{\bar{X}} u d x_{1}\right) \\
& -\sum_{j, k \neq 1} \xi_{j} \xi_{k} \frac{\partial}{\partial \delta^{k}} \int_{0}^{\bar{X}} f^{j}(u) d x_{1} \\
& +i \bar{X} \xi_{1} \lambda\left(\hat{L} \frac{\partial u}{\partial s}\right)(0)+\bar{X} \xi_{1} \sum_{j \neq 1} \xi_{j}\left(\hat{L} \frac{\partial u}{\partial \delta^{j}}\right)(0)
\end{aligned}
$$

is a homogeneous degree two polynomial, and

$$
C^{\ell}(\xi, \lambda):=\lambda\left[\hat{L} w_{\lambda}^{l}\right]+\sum_{j \neq 1} \xi_{j}\left[\hat{L} w_{\xi_{j}}^{l}\right]-i \bar{X} \xi_{1} \hat{L} w^{l}(0)
$$

are homogeneous degree one polynomials given by

$$
\frac{\partial}{\partial a_{j}}\left(\lambda \int_{0}^{\bar{X}} u\left(x_{1}\right) d x_{1}+\sum_{j \neq 1} \xi_{j} \int_{0}^{\bar{X}} i f^{j}\left(u\left(x_{1}\right)\right) d x_{1}-i \bar{X} \xi_{1}\left(u^{\prime}(0)-f^{1}(u(0))\right)\right)
$$


for $\ell=j=2, \ldots, n$, and

$$
\frac{\partial}{\partial q_{j}}\left(\lambda \int_{0}^{\bar{X}} u\left(x_{1}\right) d x_{1}+\sum_{j \neq 1} \xi_{j} \int_{0}^{\bar{X}} i f^{j}\left(u\left(x_{1}\right)\right) d x_{1}-i \bar{X} \xi_{1}\left(u^{\prime}(0)-f^{1}(u(0))\right)\right)
$$

for $\ell=n+j=n+1, \ldots, 2 n$.

Thus, the leading order part of $D$ near $(\xi, \lambda)=(0,0)$ is the homogeneous degree $(n+1)$ polynomial

$$
\Delta_{1}(\xi, \lambda):=\Gamma_{0} \operatorname{det}\left(\begin{array}{ccc}
c(\xi, \lambda) & {\left[w^{2}(0,0)\right]} & \cdots \\
C(\xi, \lambda) & C^{2}(\xi, \lambda) & \ldots
\end{array}\right)
$$

with $c, C, C^{\ell}$ defined as above. In particular, the Evans function has a zero of order $n+1$ at $(\lambda, \xi)=(0,0)$.

\section{Slow modulation approximation}

Next, we carry out a multi-dimensional version of the slow modulation (WKB) expansion in [8]. Rescale $(x, t) \mapsto(\epsilon x, \epsilon t)$ in (1) to obtain

$$
u_{t}+\sum_{j} f^{j}(u)_{x_{j}}=\epsilon \sum_{j, k}\left(B^{j k}(u) u_{x_{k}}\right)_{x_{j}} .
$$

Let

$$
u^{\epsilon}(x, t)=u^{0}\left(x, t, \frac{\phi(x, t)}{\epsilon}\right)+\epsilon u^{1}\left(x, t, \frac{\phi(x, t)}{\epsilon}\right)+\cdots,
$$

where $y \mapsto u^{0}(x, t, y)$ is a periodic function with $\partial_{x} \phi \neq 0$. We plug (23) into (22) and consider the equations obtained by equating coefficients at successive powers of $\epsilon$. At order $\epsilon^{-1}$, we have

$$
-s \partial_{y} u^{0}+\sum_{j} \omega \nu_{j} \partial_{y}\left(f^{j}\left(u^{0}\right)\right)-\partial_{y}\left(\sum_{j, k} \omega^{2} \nu_{j} \nu_{k} B^{j k}\left(u^{0}\right) \partial_{y} u^{0}\right)=0,
$$

with

$$
s:=-\frac{\partial_{t} \phi}{\left|\partial_{x} \phi\right|}, \quad \nu:=\frac{\partial_{x} \phi}{\left|\partial_{x} \phi\right|}, \quad \omega:=\left|\partial_{x} \phi\right|,
$$

which may be recognized as the traveling-profile equation after rescaling $y \rightarrow$ $\omega y$. That is, $u^{0}(y)=\bar{u}(\omega y)$ for a periodic profile of period $X=\omega^{-1}$, hence $u^{0}$ is periodic of period one, as described in [8]. The quantities $\omega(x, t), s(x, t)$, $\nu(x, t)$ are the local frequency, speed, and direction of the modulated wave. At order $\epsilon^{0}$, we have

$$
\partial_{t} u^{0}+\sum_{j=1}^{d} \partial_{x_{j}}\left(f^{j}\left(u^{0}\right)-\sum_{k=1}^{d} B^{j k}\left(u^{0}\right) \omega \nu_{k} \partial_{y} u^{0}\right)=\partial_{y}(\ldots) .
$$


Taking the average with respect to $y$, and rescaling with $y:=\omega y$, we obtain

$$
\partial_{t} M\left(u^{0}\right)+\sum_{j=1} \partial_{x_{j}} F^{j}\left(u^{0}\right)=0
$$

where

$$
F^{j}\left(u^{0}\right)=\frac{1}{X} \int_{0}^{X}\left(f^{j}\left(u^{0}\right)-\sum_{k=1}^{d} B^{j k}\left(u^{0}\right) \omega \nu_{k} \partial_{y} u^{0}\right) d y
$$

is the averaged flux along orbit $u^{0}$ (now rescaled to actual period $\bar{X}$ ), with

$$
\sum_{j} \nu_{j} F^{j}=(S M+Q)\left(u^{0}\right)
$$

by the profile equation. In the Laplacian case $B^{j k}=\delta_{k}^{j}$, (24) simplifies to

$$
F^{j}\left(u^{0}\right)=\frac{1}{X} \int_{0}^{X}\left(f^{j}\left(u^{0}\right)-\nu_{j}\left(u^{0}\right) \partial_{y} u^{0}\right) d y .
$$

We have additional $d$ equations

$$
\partial_{t}(\Omega N)\left(u^{0}\right)+\partial_{x}\left(\Omega S\left(u^{0}\right)\right)=0
$$

from the Schwarz identity $\partial_{t} \partial_{x} \phi=\partial_{x} \partial_{t} \phi$, where $d$ is the dimension of the spatial variable $x$. (Note: $(\Omega, N)$ may be regarded as polar coordinates for $\Omega N$.)

Combining, we obtain finally the closed homogenized system

$$
\partial_{t}(M, \Omega N)+\sum_{j} \partial_{x_{j}}\left(F^{j}, \Omega S e_{j}\right)=0
$$

of the introduction, consisting of $n+d$ equations in $n+d$ unknowns. As discussed in the introduction, this should be supplemented with the constraint

$$
\operatorname{curl}(\Omega N) \equiv 0
$$

coming from the relation $\Omega N=\nabla_{x} \phi$.

\section{Proof of the main theorem}

We now carry out the proof of Theorem 1.1, restricting for readability to the Laplacian case $B^{j k}=\delta_{k}^{j}$. The general case follows similarly. We want to see that the leading order part $\Delta_{1}$ of $D$, defined in (21), is given by a (nonzero) constant multiple of $\lambda^{1-d}$ times

$$
\hat{\Delta}(\xi, \lambda)=\operatorname{det}\left(\lambda \frac{\partial(M, \Omega N)}{\partial \dot{u}}(\dot{\bar{u}})+\sum_{j} i \bar{\omega} \bar{X} \xi_{j} \frac{\partial\left(F^{j}, S \Omega e_{j}\right)}{\partial \dot{u}}(\dot{\bar{u}})\right),
$$


where $\dot{\bar{u}}$ denotes the orbit class of $\bar{u}$, with $\bar{\nu}=(1,0, \ldots, 0)=N(\dot{\bar{u}})$.

Recall the assumption (H2) and (17). The tangent space to $P$ at $\bar{u}$ is the $\beta$-projection of the kernel of

$$
Z(\beta, \delta, \gamma):=\beta^{0}\left[\frac{\partial u}{\partial s}\right]+\sum_{1}^{2 n} \beta^{l}\left[w^{l}\right]+\sum_{j=2}^{d} \delta^{j}\left[\frac{\partial u}{\partial \delta^{j}}\right]+\gamma \bar{u}^{\prime}(0)
$$

and the tangent space to $\mathcal{P}$ at $\dot{\bar{u}}$ is the $\beta$-projection of the kernel of $\mathcal{Z}\left(\beta^{0}, \beta^{2}, \ldots, \beta^{2 n}, \delta^{2}, \ldots, \delta^{d}, \gamma\right):=\beta^{0}\left[\frac{\partial u}{\partial s}\right]+\sum_{2}^{2 n} \beta^{l}\left[w^{l}\right]+\sum_{j=2}^{d} \delta^{j}\left[\frac{\partial u}{\partial \delta^{j}}\right]+\gamma \bar{u}^{\prime}(0)$.

We relabel $\beta^{1}$ for $\beta^{0}$ since we will not use $w^{1}$ hereafter. Thus,

$$
\mathcal{Z}(\beta, \delta, \gamma):=\beta^{1}\left[\frac{\partial u}{\partial s}\right]+\sum_{2}^{2 n} \beta^{l}\left[w^{l}\right]+\sum_{j=2}^{d} \delta^{j}\left[\frac{\partial u}{\partial \delta^{j}}\right]+\gamma \bar{u}^{\prime}(0)
$$

for $\beta \in \mathbb{C}^{2 n}, \delta \in \mathbb{C}^{d-1}, \gamma \in \mathbb{C}$. We easily compute (see (19) for $d Q$ ) the differentials

$$
\begin{aligned}
& d X \cdot(\beta, \delta, \gamma)=\gamma=\partial X \\
& d S \cdot(\beta, \delta, \gamma)=\beta^{1}=\partial S \\
& d N \cdot(\beta, \delta, \gamma)=(0, \delta)^{T}=\partial N \\
& d \Omega \cdot(\beta, \delta, \gamma)=-\bar{\omega}^{2} \gamma=\partial \Omega \\
& d Q \cdot(\beta, \delta, \gamma)=\left(\beta^{n+1}, \ldots, \beta^{2 n}\right)^{T}=-\sum_{2}^{2 n} \beta^{l} \hat{L}^{l}=\partial Q
\end{aligned}
$$

where, following [8], we use the notation $\partial G$ to indicate the extension to $\mathbb{C}^{2 n+d}$ of a differential $d G$ defined on the kernel of $\mathcal{Z}$. (Note: this includes the extension from real to complex values, of which we shall later make important use in parametrizations (37) and (38).)

Likewise, $X M=\int_{0}^{X} u(y) d y$ gives

$$
\begin{aligned}
d(X M) \cdot(\beta, \delta, \gamma) & \\
& =\gamma \bar{u}(0)+\beta^{1} \int_{0}^{\bar{X}} \frac{\partial u}{\partial s} d y+\sum_{2}^{2 n} \beta^{l} \int_{0}^{\bar{X}} w^{l} d y+\sum_{2}^{d} \delta^{j} \int_{0}^{\bar{X}} \frac{\partial u}{\partial \delta^{j}} d y \\
& =\partial(X M)
\end{aligned}
$$

thus determining

$$
\partial M=\bar{\omega}(\partial(X M)-\bar{M} \partial X)
$$


Finally, variations

$$
d\left(X F^{j}\right)(u)=d \int_{0}^{X}\left(f^{j}(u)-\nu_{j}(u) \partial_{y} u\right) d y=\partial\left(X F^{j}\right)(u)
$$

for $j \neq 1$ have the simple form

$$
\partial\left(X F^{j}\right)(u)=\partial X f^{j}(u)(0)+\int_{0}^{X} \partial f^{j}(u) d y,
$$

by (25) and periodicity of $u$, hence

$$
\begin{aligned}
\partial F^{j}(u) & =\omega\left(\partial\left(X F^{j}\right)-(\partial X) F^{j}(u)\right) \\
& =\omega \gamma f^{j}(u)(0)+\omega \int_{0}^{X} \partial f^{j}(u) d y-\omega \gamma F^{j}(u) .
\end{aligned}
$$

To find the variation for $F^{1}$, note that, by the first-order traveling wave system (3),

$$
\sum_{j} \nu_{j} F^{j}=M S+Q
$$

so that $\sum_{j}\left(\partial \nu_{j}\right) F^{j}+\sum_{j} \nu_{j}\left(\partial F^{j}\right)=\partial(M S+Q)$, hence, for $\nu=(1,0, \ldots, 0)$, $s=0$,

$$
\partial F^{1}(u)=\partial(M S+Q)-\sum_{j} \delta^{j} F^{j}(u)=M \partial S+\partial Q-\sum_{j \neq 1} \delta^{j} F^{j}(u)
$$

We may now compute the determinant (29), i.e., the determinant of the restriction to ker $\mathcal{Z}$ of the linear map

$$
\begin{aligned}
H_{\lambda, \xi}(\beta, \delta, \gamma) & =\left(\begin{array}{c}
H_{\lambda, \xi}^{1}(\beta, \delta, \gamma) \\
H_{\lambda, \xi}^{2}(\beta, \delta, \gamma)
\end{array}\right) \\
& :=\left(\begin{array}{c}
\lambda(\bar{\Omega} \partial N+\bar{N} \partial \Omega)+\sum_{j} i \xi_{j} \beta^{1} \bar{\Omega} e_{j} \\
\lambda \partial M+i \bar{\omega} \bar{X} \xi_{1}\left(\bar{M} \partial S+\partial Q-\sum_{j \neq 1} \delta^{j} \bar{F}^{j}\right)+\sum_{j \neq 1} i \bar{\omega} \bar{X} \xi_{j} \partial F^{j}
\end{array}\right),
\end{aligned}
$$

which can be evaluated using an ingenious trick of [8] as

$$
\operatorname{det}\left(\left.H_{\lambda, \xi}\right|_{\operatorname{ker} \mathcal{Z}}\right)=C_{1} \operatorname{det}\left(\begin{array}{c}
H_{\lambda, \xi}^{1} \\
H_{\lambda, \xi}^{2} \\
\mathcal{Z}
\end{array}\right)=\left.C_{1} C_{2}(\xi, \lambda) \operatorname{det}\left(\begin{array}{c}
H_{\lambda, \xi}^{2} \\
\mathcal{Z}
\end{array}\right)\right|_{\operatorname{ker} H^{1}(\lambda, \xi)},
$$

where $C_{1}=\operatorname{det}\left(\left.\mathcal{Z}\right|_{\operatorname{ker} \mathcal{Z}^{\perp}}\right)^{-1}$ is well-defined thanks to full rank of $\mathcal{Z}$, assumption $(\mathrm{H} 2)^{\prime}$, and independent of $(\xi, \lambda)$, by the corresponding property of $\mathcal{Z}$, but $C_{2}(\xi, \lambda)=\left.\operatorname{det} H^{1}\right|_{\operatorname{ker}\left(H^{1}\right) \perp}$ and

$$
\left.\operatorname{det}\left(\begin{array}{c}
H_{\lambda, \xi}^{2} \\
\mathcal{Z}
\end{array}\right)\right|_{\operatorname{ker} H^{1}(\lambda, \xi)}
$$


both depend on the specific dependence on $(\xi, \lambda)$ of the basis chosen for ker $H^{1}$.

Note that determinant (29) is in the first place defined only up to a constant factor depending on the parametrization of $\mathcal{P}$, so that we need only take care of the $(\xi, \lambda)$ dependence of $C_{2}$. Rewriting

$$
H_{\lambda, \xi}^{1}(\beta, \delta, \gamma)=\lambda\left(\sum_{2}^{d} \bar{\omega} \delta^{j}-\bar{\omega}^{2} \gamma e_{1}\right)+\sum_{1}^{d} i \xi_{j} \beta^{1} \bar{\omega} e_{j}=0
$$

as $i \xi_{1} \bar{\omega} \beta^{1}=\lambda \bar{\omega}^{2} \gamma$, and $\lambda \bar{\omega} \delta^{j}=-i \xi_{j} \bar{\omega} \beta^{1}, j \neq 1$, and setting as in [8]

$$
\beta^{1}=-\lambda \rho, \quad \gamma=-i \bar{X} \xi_{1} \rho
$$

$\rho \in \mathbb{C}$, giving also the (new, multi-dimensional) relations

$$
\delta^{j}=i \xi_{j} \rho,
$$

and leaving $\beta^{\ell}$ free for $\ell \neq 1$, determines a choice of basis for $\operatorname{ker} H^{1}$, for which $C_{2}(\xi, \lambda)$ has the simple form $\lambda^{d-1}$.

This fact is most easily verified by right-multiplying

$$
\operatorname{det}\left(\begin{array}{c}
H_{\lambda, \xi}^{1} \\
H_{\lambda, \xi}^{2} \\
\mathcal{Z}
\end{array}\right)
$$

by the determinant one matrix

$$
\left(\begin{array}{cccc}
0 & \alpha & \lambda \rho & 0 \\
0_{2 n-1} & 0_{2 n-1} & 0_{2 n-1} & I_{2 n-1} \\
I_{d-1} & 0_{d-1} & i \tilde{\xi} & 0_{d-1} \\
0 & \beta & i \bar{X} \xi_{1} \rho & 0
\end{array}\right)
$$

$\alpha i \bar{X} \xi_{1} \rho-\beta \lambda \rho=1$, to obtain

$$
\left(\begin{array}{cc}
N_{1} & 0_{2 n} \\
* & N_{2}
\end{array}\right), \quad N_{1}:=\left(\begin{array}{cc}
1 & 0 \\
0 & \omega \lambda I_{d-1}
\end{array}\right)
$$

where $N_{2}$ is the $2 n \times 2 n$ matrix corresponding to linear operator $\left(\begin{array}{c}H^{2} \\ \mathcal{Z}\end{array}\right)$ operating on $\left(\rho, \beta^{2}, \ldots, \beta^{2 n}\right)$ through the compositions (37) and (38), and thus

$$
\hat{\Delta}(\xi, \lambda)=C_{1} \lambda^{d-1} \operatorname{det} N_{2} .
$$

Alternatively, we may observe that $H^{1}$ is full rank whenever $\lambda \neq 0$. Observing also a posteriori that $\operatorname{det} N_{2}$ is homogeneous degree $n+1$, we may conclude

\footnotetext{
${ }^{1}$ We make the inessential change $\rho \rightarrow-\rho$ for convenience in later calculations.
} 
that $C(\xi, \lambda)=\left.\operatorname{det} H^{1}\right|_{\operatorname{ker}\left(H^{1}\right)^{\perp}}$ as the ratio of $n+d$ and $n+1$ degree homogeneous polynomials must be a constant times $\lambda^{d-1}$. This discussion repairs a minor omission in [8], where the dependence of $C_{2}$ on $(\xi, \lambda)$ is not explicitly discussed.

It remains to compute, under the compositions (37), (38), the $2 n \times 2 n$ determinant $\operatorname{det} N_{2}$, which, transposing first and second $n$-row blocks, may be expressed as

$$
\operatorname{det}\left(\begin{array}{c}
\beta^{1}\left[\frac{\partial u}{\partial s}\right]+\sum_{2}^{2 n} \beta^{l}\left[w^{l}\right]+\gamma \bar{u}^{\prime}(0)+\sum_{2}^{d} \delta^{j}\left[\frac{\partial u}{\partial \delta^{j}}\right] \\
\lambda \partial M+i \bar{\omega} \bar{X} \xi_{1}\left(\bar{M} \partial S+\partial Q-\sum_{j \neq 1} \delta^{j} \bar{F}^{j}\right)+\sum_{j \neq 1} i \bar{\omega} \bar{X} \xi_{j} \partial F^{j}
\end{array}\right) .
$$

Substituting from (37), (38) and the variational formulae (30)-(36), and expressing $N_{2}$ as a matrix taking $\left(\rho, \beta^{2}, \ldots, \beta^{2 n}\right) \rightarrow \mathbb{C}^{2 n}$, we obtain, similarly as in [8], that $\operatorname{det} N_{2}=\hat{\Delta} \lambda^{1-d}$ is $\bar{\omega}^{n}$ times the determinant $\Delta_{1}$ defined in (21), giving the desired relation $\Delta_{1}=\bar{\omega}^{-n} \lambda^{1-d} \hat{\Delta}$, and completing the proof.

Namely, the first line of (39) becomes

$$
-\lambda \rho\left[\frac{\partial u}{\partial s}\right]+\sum_{2}^{2 n} \beta^{l}\left[w^{l}\right]-i \bar{X} \xi_{1} \rho \bar{u}^{\prime}(0)+\sum_{2}^{d} i \xi_{j} \rho\left[\frac{\partial u}{\partial \delta^{j}}\right] .
$$

The second line of (39) becomes

$$
\begin{aligned}
& \lambda \bar{\omega}\left(\gamma \bar{u}(0)+\beta^{1} \int_{0}^{\bar{X}} \frac{\partial u}{\partial s} d x_{1}+\sum_{2}^{2 n} \beta^{l} \int_{0}^{\bar{X}} w^{l} d x_{1}+\sum_{2}^{d} \delta^{j} \int_{0}^{\bar{X}} \frac{\partial u}{\partial \delta^{j}} d x_{1}-\bar{M} \gamma\right) \\
& +i \bar{\omega} \bar{X} \xi_{1}\left(\bar{M} \beta^{1}-\sum_{2}^{2 n} \beta^{l} \hat{L} w^{l}-\sum_{2}^{d} \delta^{j} F^{j}(\bar{u})\right) \\
& +\sum_{j \neq 1} i \bar{\omega} \bar{X} \xi_{j}\left(\bar{\omega} \gamma f^{j}(\bar{u})(0)+\bar{\omega} \int_{0}^{\bar{X}} \partial f^{j}(\bar{u}) d x_{1}-\bar{\omega} \gamma F^{j}(\bar{u})\right) \\
& =\lambda \bar{\omega}\left(-i \bar{X} \xi_{1} \rho \bar{u}(0)-\lambda \rho \int_{0}^{\bar{X}} \frac{\partial u}{\partial s} d x_{1}+\sum_{2}^{2 n} \beta^{l} \int_{0}^{\bar{X}} w^{l} d x_{1}\right. \\
& \left.+\sum_{2}^{d} i \xi_{j} \rho \int_{0}^{\bar{X}} \frac{\partial u}{\partial \delta^{j}} d x_{1}+\bar{M} i \bar{X} \xi_{1} \rho\right) \\
& +i \bar{\omega} \bar{X} \xi_{1}\left(-\bar{M} \lambda \rho-\sum_{2}^{2 n} \beta^{l} \hat{L} w^{l}-\sum_{2}^{d} i \xi_{j} \rho F^{j}(\bar{u})\right) \\
& +\sum_{j \neq 1} i \bar{\omega} \bar{X} \xi_{j}\left(-\bar{\omega} i \bar{X} \xi_{1} \rho f^{j}(\bar{u})(0)+\bar{\omega} \int_{0}^{\bar{X}} \partial f^{j}(\bar{u}) d x_{1}+\bar{\omega} i \bar{X} \xi_{1} \rho F^{j}(\bar{u})\right)
\end{aligned}
$$




$$
\begin{aligned}
= & -\bar{\omega} \rho\left(\lambda i \bar{X} \xi_{1} \bar{u}(0)+\lambda^{2} \int_{0}^{\bar{X}} \frac{\partial u}{\partial s} d x_{1}-\lambda i \sum_{2}^{d} \xi_{j} \int_{0}^{\bar{X}} \frac{\partial u}{\partial \delta^{j}} d x_{1}\right. \\
& \left.-\bar{X} \xi_{1} \sum_{2}^{d} \xi_{j} f^{j}(\bar{u})(0)\right)+\bar{\omega} \sum_{2}^{2 n} \beta^{l}\left(\lambda \int_{0}^{\bar{X}} w^{l} d x_{1}-i \bar{X} \xi_{1} \hat{L} w^{l}\right) \\
& +\bar{\omega} i \sum_{2}^{d} \xi_{j} \int_{0}^{\bar{X}} \partial f^{j}(\bar{u}) d x_{1} \\
= & -\bar{\omega} \rho\left(-\lambda i \bar{X} \xi_{1} \hat{L}\left(\frac{\partial u}{\partial s}\right)(0)+\lambda^{2} \int_{0}^{\bar{X}} \frac{\partial u}{\partial s} d x_{1}-\lambda i \sum_{2}^{d} \xi_{j} \int_{0}^{\bar{X}} \frac{\partial u}{\partial \delta^{j}} d x_{1}\right. \\
& \left.-\bar{X} \xi_{1} \sum_{2}^{d} \xi_{j} \hat{L}\left(\frac{\partial u}{\partial \delta^{j}}\right)(0)\right)+\bar{\omega} \sum_{2}^{2 n} \beta^{l}\left(\lambda \int_{0}^{\bar{X}} w^{l} d x_{1}-i \bar{X} \xi_{1} \hat{L} w^{l}\right) \\
& +\bar{\omega} i \sum_{2}^{d} \xi_{j}\left(-\lambda \rho \int_{0}^{\bar{X}} \frac{\partial f^{j}}{\partial u} \frac{\partial u}{\partial s} d x_{1}+\sum_{2}^{2 n} \beta^{l} \int_{0}^{\bar{X}} A^{j} w^{l} d x_{1}\right. \\
& \left.+\sum_{k=2}^{d} i \xi_{k} \rho \int_{0}^{\bar{X}} \frac{\partial f^{j}}{\partial u} \frac{\partial u}{\partial \delta^{k}} d x_{1}\right)
\end{aligned}
$$

with (32) and other identities. Denoting by $\mathcal{N}$ the matrix in (21) for which $\Delta_{1}=\operatorname{det} \mathcal{N}$, we find, comparing term by term, that the first $n$ rows of $N_{2}$ are equal to the first $n$ rows of $\mathcal{N}$, while the last $n$ rows of $N_{2}$ are equal to $\bar{\omega}$ times the last $n$ rows of $\mathcal{N}$. Thus, $\operatorname{det} N_{2}=\bar{\omega}^{n} \operatorname{det} \mathcal{N}=\bar{\omega}^{n} \Delta_{1}$ as claimed, and we are done.

Acknowledgement. K.Z. thanks B. Texier for his interest in the problem, and for several stimulating conversations. Research of the authors was supported in part by the National Science Foundation under Grants No. DMS-0204072 (M.O.) and DMS-0300487 (K.Z.).

\section{References}

[1] Gardner, R., On the structure of the spectra of periodic traveling waves. $J$. Math. Pures Appl. 72 (1993), 415 - 439.

[2] Gues, O., Metivier, G., Williams, M., and Zumbrun, K., Multidimensional viscous shocks I: Degenerate symmetrizers and long time stability. J. Amer. Math. Soc. 18 (2005), $61-120$.

[3] Guès, O., Métivier, G., Williams, M., and Zumbrun, K., Multidimensional viscous shocks II: The small viscosity problem. Comm. Pure Appl. Math. 57 (2004), $141-218$. 
[4] Guès, O., Métivier, G., Williams, M., and Zumbrun, K., Existence and stability of multidimensional shock fronts in the vanishing viscosity limit. Arch. Rational Mech. Anal. 175 (2005), $151-244$.

[5] Metivier, G. and Zumbrun, K., Large Viscous Boundary Layers for Noncharacteristic Nonlinear Hyperbolic Problems. Mem. Amer. Math. Soc. 175 (2005), no. 826 .

[6] Oh, M. and Zumbrun, K., Stability of periodic solutions of viscous conservation laws with viscosity - 1. Analysis of the Evans function, Arch. Rational Mech. Anal. 166 (2003), 99 - 166.

[7] Oh, M. and Zumbrun, K., Stability of periodic solutions of viscous conservation laws with viscosity - 2. Pointwise bounds on the Green function. Arch. Rational Mech. Anal. 166 (2003), $167-196$.

[8] Serre, D., Spectral stability of periodic solutions of viscous conservation laws: Large wavelength analysis. Comm. Partial Differential Equations 30 (2005)(13), $259-282$.

[9] Serre, D., Entropie du mélange liquide-vapeur d'un fluide thermo-capillaire. Arch. Rational Mech. Anal. 128 (1994), 33 - 73.

[10] Serre, D., Private Communicaton.

[11] Zumbrun, K., Multidimensional Stability of Planar Viscous Shock Waves (TMR Summer School Lectures, Kochel am See, May 1999; eds: H. Freistüeler and A. Szepessy). Progress in Nonlinear Differential Equations and their Applications 47. Boston: Birkhäuser 2002, pp. $307-516$.

[12] Zumbrun, K. and Serre, D., Viscous and inviscid stability of multidimensional planar shock fronts. Indiana Univ. Math. J. 48 (1999), 937 - 992.

Received October 16, 2005 